\title{
Study on Algorithms of Power Line Extraction Based on Power Line Image Feature
}

\author{
Wen-Yu WANG ${ }^{1, a,{ }^{*}}$, Yuan-Jing LIU ${ }^{2, b}$, Le ZHAO ${ }^{2, c}$, Xian-Pei WANG ${ }^{2, d}$ \\ ${ }^{1}$ School of Journalism and Communication, Wuhan Sports University, Wuhan 430073, Hubei \\ Province, China \\ ${ }^{2}$ Electronic Information School, Wuhan University, Wuhan 430072, Hubei Province, China \\ a405424059@qq.com,b2419795725@qq.com,, ${ }^{\text {zhaole@whu.edu.cn, }{ }^{d} x p w a n g @ w h u . e d u . c n ~}$ \\ "Corresponding author
}

Keywords: UAV, Power Line, Extraction, Algorithms.

\begin{abstract}
Using small commercial UAV equipped with a simple visible light camera, to obtain the depth information of power line and the ground by monocular or binocular vision is the focus of research. Among them, how to use digital image processing method to extract the power line quickly, accurately, automatically is one of the core issues. This paper analyzes the Hough transform, Radon transform and LSD algorithm in terms ofthe effect of power line extraction by contrast. The problems of the three algorithms are pointed out, a kind of Power line extraction algorithm based on image feature of power line is put forward, in order to increase the accuracy rate of power line detection. Relative experiments are done to verity the effectiveness of algorithm.
\end{abstract}

\section{Summary of Line Extraction}

To extract the characteristics of the straight line is a very important linkto identify the characteristics of the target, it has been the focus of many experts' research[1]. The commonly used linear extraction methods generally can be divided into the following three categories[2-5], the first category is the line extraction method based on transform domain, which is performed in the transform domain space. Through the relationship between transform domain and image domain, the straight line is locatedby inverse transform, the classical algorithms are Hough transform and Radon transform; the second category is based on gradient.Combingthe amplitude and direction of gradient, the straight line is extractedby"edge region growing", such as Line Segment Detector (LSD), Phase grouping etc.; the third category is line extraction method based on chain code. Use the linear feature of digital image to track the edge chain code and realize the line extractionbaccording to the characteristics of chain code[6].In this paper,the effect of power line extraction of Hough transform, Radon transform and LSD algorithm are compared and analyzed, problems are pointed out and a kind of algorithm which restraints on power line extraction range using a priori knowledge is put forward, in order to increase the accuracy rate of power line detection. Related experiments show that this algorithm is effective.

\section{Classical Line Extraction Algorithm}

Fig.1 shows threegraphs of small UAV monocular acquisition. In the MATLAB programming environment, this paper uses MATLAB's Sobel algorithm as method of edge detection. Fig. 2 is the extraction result of Hough transform, Fig. 3 is the extraction result of Radon transform, Fig.4 is the extraction result of LSD transform. 


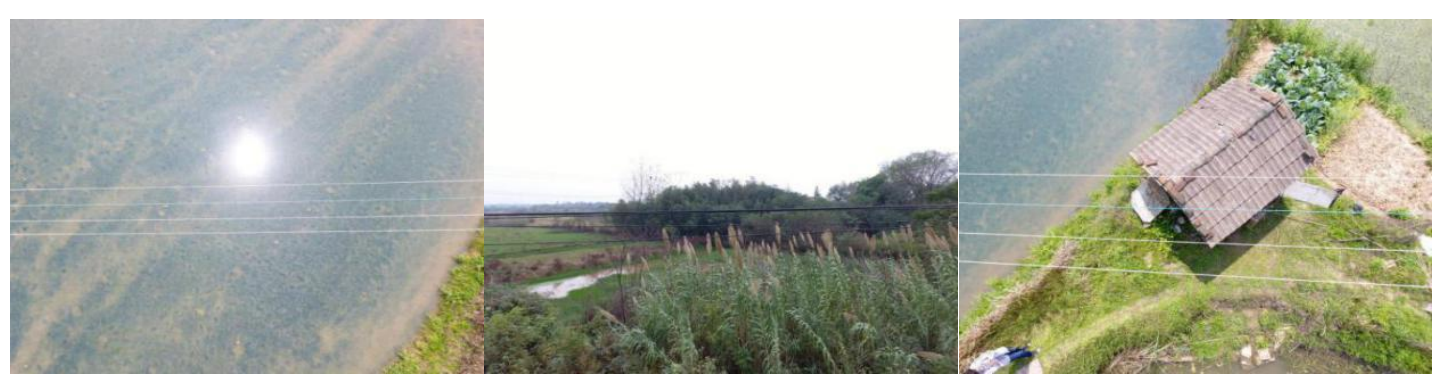

(a) Simple background(b) Tree as background(c) House \&farm as background

Fig.1.The three types of original image

Although Hough transform shows better anti-noise ability in line detection, but in a more complex background, such as farmland, weed interference under the trees, its performance reduces greatly and sometimes the power linecan notbe detected completely, otherwise, some irrelevant segments are checked out, such as house ridge, weeds, road and so on. Radon transform also showsgood anti-noise ability even if it is in the complex background. However, the classical Radon transform can only obtain the linear direction and radial coordinate, it is unable to determine the location coordinate, the starting point, end point of the line segment, and line width information. As can be seen from the results of LSD, it shows great performance in extracting details, even if the power line is weak and has better performance than Hough transform in terms of the error rate of extraction, and can better locates the power line.But error detection can be caused when the background is complex. Therefore, image characteristics of power line are studied to make further improvement.

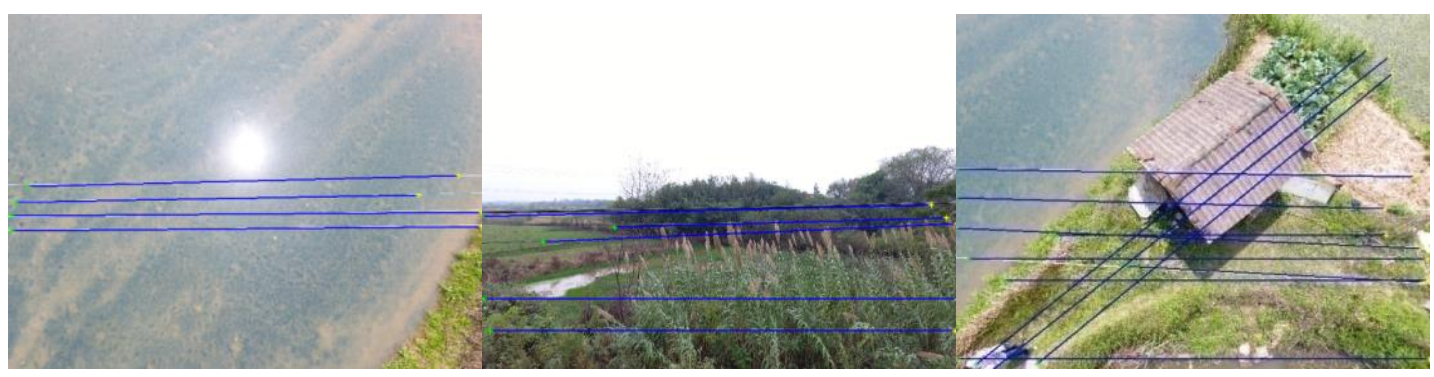

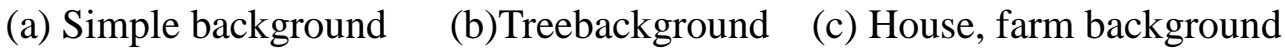

Fig. 2. Results of Hough transform extraction

\section{Power Line Extraction Algorithm Based on Power Line Image Features}

\section{Radon Transform Based on Angle and Distance Constraint}

Although the power line extraction range has made certain constraints and achieved good results, but in this range disruptors also exists. In addition, the power lines have a certain pixel width, more straight lines can be detected at the position of one power line, as shown in Fig. 5.Aiming at this phenomenon, this paper refers to the extraction method of tracks and proposes a constraint based on the distance and the angle of inclination between two power lines. This condition is based on the fact that a set of power lines is approximately parallel in the image, or it can be said that in the images the two power lines show smaller angle and maintain a certain distance. 


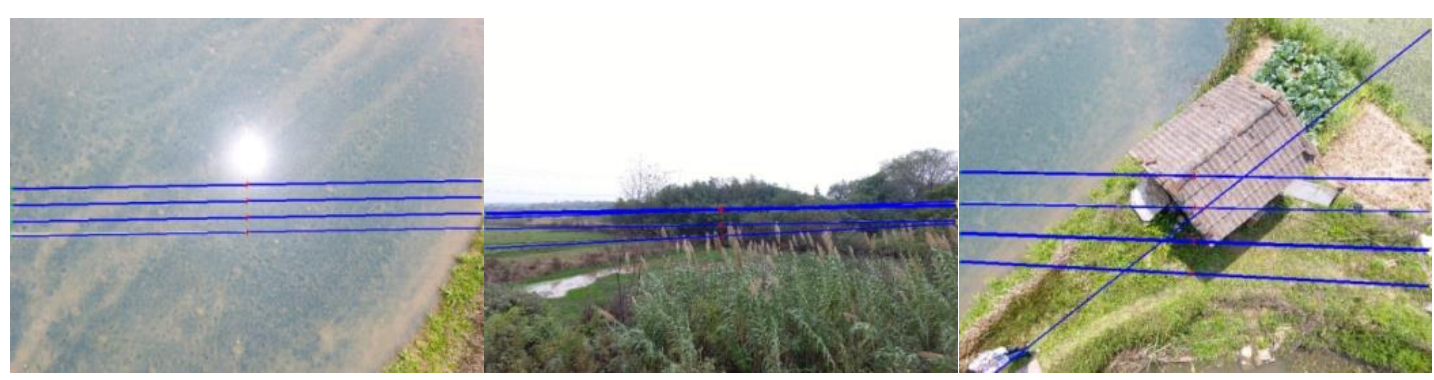

(a) simple background (b)treebackground $\quad$ (c) house,farm background

Fig.3.Extraction result of Radon transform

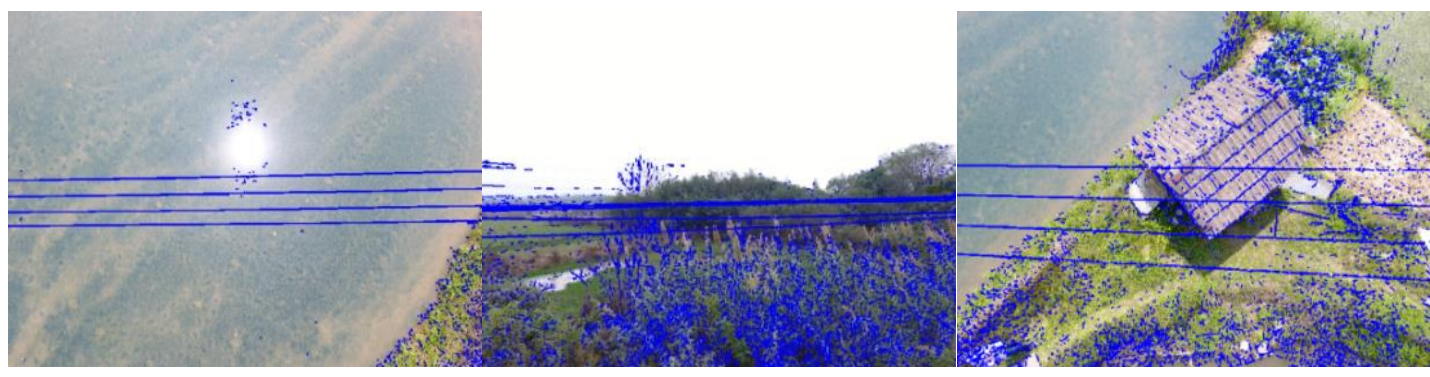

(a) simple background (b)treebackground (c) house,farm background

Fig. 4.Extraction result ofLSD

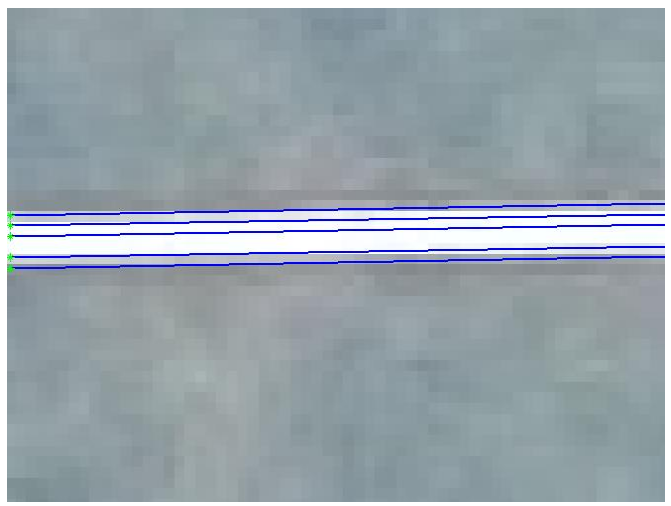

Fig.5.Repeated extraction of Radon transform

The basic thought of the method:under the condition that the extreme point information of the Radon transform has been obtained, the other lines with larger angle difference are screened out, and the straight lines having approximately the same radius value are classified to the same group.

The flow of the method is:

(1) the information of all extreme points is obtained by means of Radon transform, and the lines with approximately the same inclination angle and the radial coordinate are divided into the same group, and an extreme point of each group is taken out as the representative point of this group, which represents this line.

(2) the representative points, $n$ is sorted outin the sequence of smallto big, we can get $\theta_{1}<\theta_{2} \ldots<\theta_{\mathrm{n}}, \quad n$ is the number of representative points.

(3) firstof all, when $n=1$, if $\theta_{n+1}-\theta_{n}<\theta_{t h}$ ( $\theta_{t h}$ is thresholdgreater than 0 ), save $\theta_{n}$ 's corresponding straight line, and draw the line, otherwise go to step 4.

(4) let $n=n+1$,repeat step 3 until all the representative points are traversed. 


\section{LSD Line Detection Based on Least Squares Method(LSM)}

Although LSD's error rate is low and has great performance. But in the process of line verification, part of the complete power line are cut off, leading to the discontinuity of power line detection. Therefore this paper proposes a method of grouping the line segments. The segments with isotropic features are divided into the same group, and then the least squares method are used to realize the power line fitting.

The flow of the method is:

(1) On the basis of LSD line detection, calculate the slope of all fault segments $k(i)$ and nodal increment $b(i), i=1,2,3, \ldots n, n$ isthe total number of lines detected

(2) Divide the lineswith isotropic features into the same group.

(3) Calculate the number of the lines with isotropic features in each group $n_{1}$, find the maximum number $n_{\max }$, the lines that the number of the line segments in a group less than threshold* $n_{\max }$ aren't involved in the final line fitting.

(4) Use the least square method to fit the power line.

\section{Experiments and Results}

To verify the feasibility of extracting power line quickly and accurately with this passage's optimized Radon transform and LSD method based on Least Square, experiments are designed to extract the power lines in different background(Fig. 5(a)(b)(c)), and the running time of the whole process is recorded.The use of Radon transform in this paper is based on the Sobel and LSD method based on Least Squares, the results are shown in Fig. 6, and Fig. 7, the time efficiency is recorded in Table 1.

In regard to detection effect,the two methods proposed in this paper can both extract the power line from complex background successfullyand achievesatisfactory results. The optimized Radon transform can not only remove other interference effectively, but also avoid the shortcoming of Radon transform of extract the same power line repeatedly.The LSD method based on the Least SquaresMethod can also identify the power line accurately, and the location accuracy of this method is higher than that of the Radon transform.

In terms of time efficiency, the two methods can quickly recognize the power lineeven if aerial images are of high resolution.The optimized Radon transform is more stable in time consuming, while the time efficiency of LSD algorithm based on Least Squares can be affectedby power line image with complex background. But the extraction rate of both methods achieved satisfactory results.

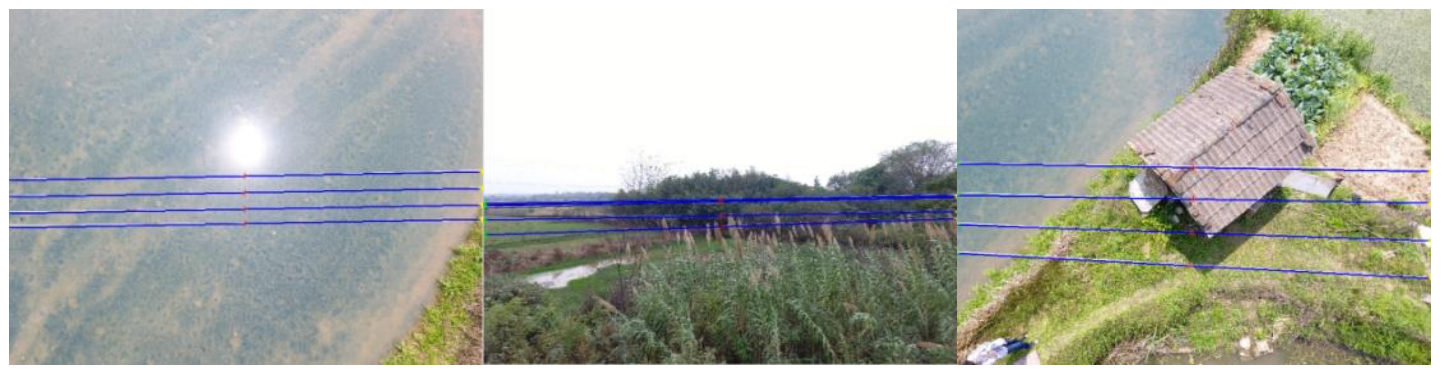

(a)Simple background (b)Tree background (c)Farm; house background

Fig.6.Extraction results with Radon transform after angle and distance constraint 


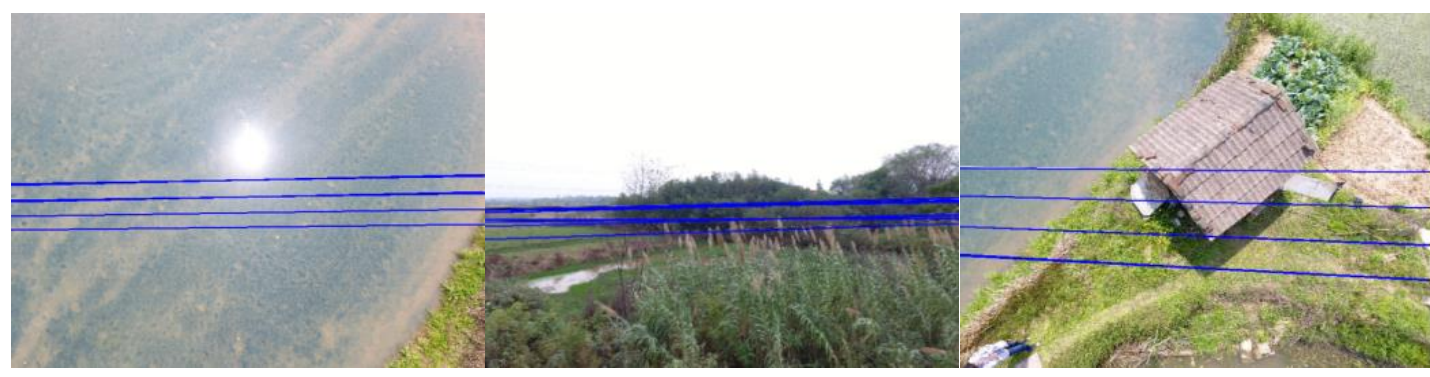

(a)Simple background(b)Tree background (c)Farm;house background

Fig.7.LSD detection result based on Least Squares Method

Table 1. Time efficiency of power line extraction in two ways

\begin{tabular}{|c|c|c|c|}
\hline Algorithm & Simple background & Tree background & $\begin{array}{c}\text { Farm, house } \\
\text { background }\end{array}$ \\
\hline Radon+ constraint & 4.6313 & 5.2813 & 5.4688 \\
\hline LSD/LSM & 2.3125 & 6.9623 & 7.5673 \\
\hline
\end{tabular}

\section{References}

[1] Liu Yuanjing. Overhead line surrounding environment analysis [D] based on image processing. Wuhan University,2017.

[2] Li Cailing, FengZhaohui, Deng Xiaojing etc. Power line extraction method in complex terrain background [J]. Computer engineering and Application.2016(22): 198-202.

[3] Chen Y, Li Y, Zhang H, et al. Automatic power line extraction from high resolution remote sensing imagery based on an improved Radon transform[J]. Pattern Recognition. 2016, 49: 174-186.

[4] Zhao Zhenbing, Wang Qin, Gao Qiang. Power line image analysis and extraction [J] using improved phase congruency detection method. High voltage technology.2011(08): 2004-2009.

[5] Von Gioi R G, Jakubowicz J, Morel J M, et al. LSD: A fast line segment detector with a false detection control [J]. IEEE Transactions on Pattern Analysis and Machine Intelligence, 2010, 32(4): $722-732$.

[6] Zhao Like, Song Weidong, Wang Jingxue. Freeman chain code priority linear extraction algorithm [J]. Journal of Wuhan University (Information Science Edition).2014(01): 42-46. 